\title{
Molecular mechanisms of statin intolerance
}

\author{
Anna Gluba-Brzozka ${ }^{1,2}$, Beata Franczyk ${ }^{3}$, Peter P. Toth ${ }^{4}$, Jacek Rysz ${ }^{2,3}$, Maciej Banach²,5
}

\author{
${ }^{1}$ Department of Nephrology, Hypertension and Family Medicine, WAM University \\ Hospital, Lodz, Poland \\ ${ }^{2}$ Healthy Aging Research Center, Medical University of Lodz, Lodz, Poland \\ ${ }^{3}$ Department of Nephrology, Hypertension and Family Medicine, Medical University \\ of Lodz, Lodz, Poland \\ ${ }^{4} \mathrm{CGH}$ Medical Center, Sterling, Illinois, and Ciccarone Center for the Prevention of \\ Cardiovascular Disease, Johns Hopkins University School of Medicine, Baltimore, \\ Maryland, USA \\ ${ }^{5}$ Department of Hypertension, Medical University of Lodz, Lodz, Poland
}

Submitted: 21 March 2016

Accepted: 29 March 2016

Arch Med Sci 2016; 12, 3: 645-658

DOI: 10.5114/aoms.2016.59938

Copyright (c) 2016 Termedia \& Banach

\author{
Corresponding author: \\ Anna Gluba-Brzozka MD, PhD \\ Department of Nephrology, \\ Hypertension and \\ Family Medicine \\ WAM University Hospital \\ 113 Żeromskiego St \\ 90-549 Lodz, Poland \\ Phone: +48 693439386 \\ E-mail: aniagluba@yahoo.pl
}

\begin{abstract}
Statins reduce cardiovascular morbidity and mortality in primary and secondary prevention. Despite their efficacy, many persons are unable to tolerate statins due to adverse events such as hepatotoxicity and myalgia/ myopathy. In the case of most patients, it seems that mild-to-moderate abnormalities in liver and muscle enzymes are not serious adverse effects and do not outweigh the benefits of coronary heart disease risk reduction. The risk for mortality or permanent organ damage ascribed to statin use is very small and limited to cases of myopathy and rhabdomyolysis. Statin-induced muscle-related adverse events comprise a highly heterogeneous clinical disorder with numerous, complex etiologies and a variety of genetic backgrounds. Every patient who presents with statin-related side effects cannot undergo the type of exhaustive molecular characterization that would include all of these mechanisms. Frequently the only solution is to either discontinue statin therapy/reduce the dose or attempt intermittent dosing strategies at a low dose.
\end{abstract}

Key words: statin intolerance, mechanisms, myalgia, myopathy, adverse effects.

\section{Introduction}

The statins reduce cardiovascular morbidity and mortality in both the primary and secondary prevention settings. Despite their efficacy, a significant number of persons throughout the world are unable to tolerate statins due to such adverse events as hepatotoxicity and myalgia/myopathy. According to a National Lipid Association Statin Intolerance Panel, statin intolerance is defined as: "adverse symptoms, signs, or laboratory abnormalities attributed by the patient (or provider) to the statin and in most cases perceived by the patient to interfere unacceptably with activities of daily living (such as sleep, work/housework, or leisure-time activity), leading to a decision to stop or reduce statin therapy" [1]. According to a position paper from an International Lipid Expert Panel, statin intolerance is: "the inability to tolerate a dose of statin required to reduce a person's CV risk sufficiently from their baseline risk and could result 
from different statin related side effects including: muscle symptoms, headache, sleep disorders, dyspepsia, nausea, rash, alopecia, erectile dysfunction, gynecomastia, and/or arthritis" [2, 3].

In the case of most patients it seems that mild-to-moderate abnormalities in liver and muscle enzymes, as well as diabetes risk, are not serious adverse effects and these potential risks of laboratory abnormalities do not outweigh the benefits of proven coronary heart disease risk reduction [1]. The risk of mortality or permanent organ damage ascribed to adverse effects of statins is very small and is limited mainly to cases of myopathy and rhabdomyolysis.

\section{Symptoms of statin intolerance}

Statin-related myopathy is diagnosed when the following criteria are met: (a) muscle symptoms appeared during statin therapy, (b) these symptoms normalized when patients received placebo, (c) muscle toxicity occurred again after re-exposure to the statin, (d) adverse effects were confirmed in pathologic and biochemical tests, and finally (e) pathologic abnormalities reversed after the discontinuation of statin therapy $[4,5]$. However, the persistence or worsening of muscle symptoms after cessation of the statin has occasionally been reported $[6,7]$. Among the most frequent symptoms of statin intolerance is myalgia unaccompanied by significant creatine kinase (CK) elevations and myositis (CK > 10 times above the upper limit of normal (ULN)) or rhabdomyolysis (CK level > 10,000 IU/I or accompanied by substantial elevation in serum creatinine level) [8]. A study by Schalke et al. [9] demonstrated that statins may also induce inflammatory myopathies (i.e. polymyositis and dermatomyositis) characterized by significant elevations of CK levels, a myopathic pattern on the electromyogram, and inflammatory infiltrates evident on muscle biopsy. However, such cases are rare, and they can be reversed by the discontinuation of statin therapy and immunosuppressive therapy (e.g., glucocorticoids) [9]. Necrotizing myopathy observed in muscle biopsies without significant inflammation occurs rarely. In these patients elevated CK levels and proximal muscle weakness were seen and autoimmune activation with myocellular injury was suggested [10, 11].

According to estimates, $3 \%$ to $20 \%$ of statin users experience statin-associated side-effects [12]. The frequency of myopathy in randomized controlled trials is estimated to be about 1.5-5.0\% [13]. However, this value is an underestimate because patients with baseline myalgia or a history of myopathy are excluded from randomization. In the Prediction of Muscular Risk in Observational Conditions (PRIMO) study [14] the frequency of muscle symptoms in outpatients receiving high-dosage statin was reported to be about $10.5 \%$ but varied by statin, with fluvastatin having the lowest frequency and simvastatin having the highest frequency of myalgia. According to a review of 21 clinical trials, the occurrence of statin-associated symptoms such as muscle pain, myopathy, and rhabdomyolysis is estimated to be 190, 5 and 1.6 per 100,000 patient years, respectively $[15,16]$. The National Health and Nutrition Examination Survey revealed that the unadjusted prevalence of musculoskeletal pain in adults aged $\geq 40$ years was significantly higher for statin users in comparison to those not treated with statins ( $23 \%$ vs. $18 \% ; p=0.02$ ). Apart from the aforementioned side effects of statins, in some cases hepatotoxicity, peripheral neuropathy, cognitive decline, tendinitis, arthritis, diabetes, insomnia, arthralgia and cataract may also appear [17]. Statin use might also be associated with an increased risk of sleep disturbances, e.g. insomnia [18]. Besides muscle symptoms, statin-intolerant patients experience other side effects including hair loss, gastroenterological disorders, joint pains, peripheral neuropathy, pseudo-lupus syndrome, sexual function problems and weight change [19-21]. According to the PRIMO study, the strongest independent risk factors for muscle symptoms are the following: a history of myopathy associated with another lipid-lowering therapy (OD = 10.12), a history of unexplained cramps $(O D=4.14)$, a history of CK elevation (OD = 2.04), a family history of muscle symptoms $(\mathrm{OD}=1.93)$, myalgia during the treatment with lipid-lowering therapy (OD = $1.89)$, and untreated hypothyroidism $(O D=1.71)$ $[8,14]$. Of interest, the use of antidepressants was associated with a considerably lower frequency of muscle symptoms $(O D=0.51)[8,14]$. Mancini et al. [17] considered female gender to be a myopathy risk factor. Other risk factors associated with myalgia and myopathy include advanced age ( $>$ 80), alcoholism, low body mass index, low muscle mass and frailty, untreated hypothyroidism, parathyroid dysfunction, consumption of large quantities of grapefruit juice (in patients taking statins with dependence on cytochrome P450 3A4 metabolism), major surgery (perioperative period), multisystem diseases (mainly related to the liver and/or kidney), and excessive physical activity [17]. Among the most important etiologies of statin adverse events are drug interactions [22]. Muscle symptoms usually appear within weeks to months but may occur at any time during therapy.

Statin therapy is sometimes associated with asymptomatic elevation of aspartate aminotransferase (AST) and alanine aminotransferase (ALT), or 'transaminitis' $[23,24]$. The frequency of hepatic biochemical abnormalities during statin therapy 
is estimated to be 1-3\%, and they appear on average 4 weeks (range 1 to 8 weeks) after the initiation of treatment $[25,26]$. AST and ALT elevations seem to be dose-related and usually transient.

A meta-analysis performed by Davidson et al. [27] demonstrated that treatment with atorvastatin (80 mg/day) and simvastatin ( $80 \mathrm{mg} /$ day) is associated with a persistent elevation of ALT ( $>3$ times ULN) up to 5-fold in comparison with atorvastatin $10 \mathrm{mg}$ and simvastatin $20-40 \mathrm{mg}(0.2 \%$ vs. $1.0 \%)$. The mechanism of hepatocellular injury is unclear. The results of animal studies have suggested that the reduction of mevalonate or one of its sterol metabolites related to the inhibition of 3-hydroxyl, 3-methyl-glutaryl-CoA reductase (HMG-CoA), may be associated with elevation of liver enzymes [28]. Also, asymptomatic elevation in ALT without other signs of ongoing liver disease or injury including a lack of histopathological changes may appear in the course of statin therapy [29]. The underlying mechanism remains unclear, but it has been suggested that transaminitis may result from changes in lipid components of hepatocyte membrane, leading to an increase in its permeability and in consequence to the "leakage" of liver enzymes without hepatotoxic consequences [30]. Moreover, it was suggested that the degree of lipophilicity of the statins may influence the occurrence of aminotransferase elevations. According to Dale et al. [31], less lipophilic statins such as rosuvastatin, pravastatin, atorvastatin, and fluvastatin raise the relative risk of aminotransferase elevation in comparison to more lipophilic ones including lovastatin, simvastatin, and cerivastatin; this is in contrast to CK elevation, which is more frequent in cases of less lipophilic statins [32]. More work in this area needs to be done to better define the role of lipophilicity/ hydrophilicity in determining the risk of adverse events with statins.

\section{Mechanisms of statin intolerance}

Although precise mechanisms of statin myopathy are under active investigation, there are several hypotheses concerning this issue. Side-effects of statins may be aggravated by concomitant use of medications increasing statin concentrations (e.g. reducing statin metabolism) [33]. Polymorphisms in the following genes correlate with increased risk of myopathy: solute carrier organic anion transporter family member 1B1 (SLCO1B1), cytochrome P450 isoenzymes, coenzyme Q, carnitine palmitoyl transferase 2, ATP-binding cassette sub-family $B$ (MDR/TAP) member 1 (ABCB1) and ATP-binding cassette subfamily $G$ member 2 (ABCG2) [8]. Statin intolerance can be ascribed to two types of mechanisms: increased bioavailability (including defects in myocyte duplication, autoimmune reaction - anti-HMG-CoA reductase antibodies, vitamin D insufficiency, and myocyte membrane instability and disruption) and genetic predisposition (mitochondrial dysfunction, decrease in mevalonate pathways products: ubiquitin - coenzyme $Q_{10}$, alteration in gene expression - apoptosis and protein degradation) [34]. The following etiologies may be associated with the development of statin myopathy: reduced production of ubiquinone or coenzyme $Q_{10}$ [35], failure to replace damaged muscle protein via the ubiquitin pathway [36], reduced sarcolemmal or sarcoplasmic reticular cholesterol [10, 37, 38], increased uptake of cholesterol or phytosterols [39, 40], diminished production of prenylated proteins $[10,37]$, disruption of calcium metabolism in the skeletal muscle [41], alterations of fat metabolism [42] and inhibition of selenoprotein synthesis [41, $43,44]$. Moreover, the induction of skeletal muscle fiber apoptosis, mitochondrial dysfunction, and terpenoid depletion may mediate statin-associated myopathy [45]. The analysis of gene expression (GeneChips, Affymetrix) in biopsies of both human vastus lateralis muscles performed $8 \mathrm{~h}$ after eccentric exercise and after statin/placebo treatment revealed the involvement of the ubiquitin proteasome pathway (UPP) in skeletal muscle in response to combined exercise and statin treatment [36]. This study demonstrated transcriptional activation of $\mathrm{FBXO} 3$ and other members of the UPP in the response to statins plus exercise, but not to statins or exercise alone. Statin- and exercise-associated upregulation of $\mathrm{FBXO} 3$ probably affects the balance between protein degradation and repair. Statins may also alter the response of muscle to exercise stress by altering UPP actions, protein folding and catabolism [36]. Urso et al. [36] suggested that statin-induced instability of skeletal muscle cell membranes may be associated with reduced membrane integrity in response to a bout of eccentric exercise and the activation of proteolytic cascades in muscle, which may explain the increase in the number of genes involved in protein catabolism. Another study, which combined complementary in vitro, animal, and human experiments, provided evidence that statins induce muscle damage by the stimulation of muscle-specific E3 ubiquitin ligase atrogin-1 [46]. The regulation of atrophy-related genes including atrogin-1 promotes enhanced muscle protein degradation by the ubiquitin proteasome pathway. This study demonstrated that statin therapy resulted in increased atrogin-1 expression and increased protein breakdown in lovastatin-treated muscle cells. Moreover, experiments by Siddals et al. [47] revealed the suppression of IGF-1 signaling following statin treatment. The role of atrogin-1 in statin muscle toxicity remains unclear. Tintgnac 
et al. [48] suggested that MyoD (a transcriptional activator critical for muscle development) might be an atrogin-1 target. It is also not known whether adverse effects of statins are associated with HMG-CoA reductase inhibition. However, the fact that myopathy is induced by structurally different statins may suggest that toxicity is the result of inhibition of this enzyme. Results of animal studies on fish with knockdown of the HMG-CoA reductase gene demonstrated a muscle phenotype closely resembling that seen after statin treatment. This observation confirms that statin-induced muscle toxicity can be a result of HMG-CoA reductase inhibition [46].

\section{Vitamin D deficiency}

Vitamin D deficiency is associated with myalgia similar to that induced by statin therapy. As reported by Lee et al. [49], nearly $73 \%$ of patients with statin-induced myalgia were vitamin D insufficient $(25-\mathrm{OH} \mathrm{D}<60 \mathrm{nmol} / \mathrm{l})$, while the rest of them were severely deficient $(25-\mathrm{OH} \mathrm{D}<30 \mathrm{nmol} / \mathrm{l})$. Cessation of statin treatment accompanied by vitamin D replacement resulted in full or significant resolution of myalgia in all patients over approximately 3 months. This was not, however, a prospective, randomized, controlled study and must be interpreted with caution. High serum levels of 25-OH D may be protective against statin-induced myalgia, since statin rechallenge in patients with higher post-treatment levels (> $80 \mathrm{nmol} / \mathrm{l}$ ) was associated with lower risk of symptom re-occurrence. In a study by Ahmed et al. [50], statin-treated patients with myalgia had lower serum vitamin $D$ levels in comparison to asymptomatic patients $(28.6 \pm 13.2$ vs. $34.2 \pm 13.8 \mathrm{ng} /$ $\mathrm{ml}, p<0.0001)$. The supplementation of vitamin D (ergocalciferol 50,000 units/week for 12 weeks) in vitamin $D$ deficient, myalgic patients resulted in the increase of serum vitamin D from 20.4 \pm 7.3 to $48.2 \pm 17.9 \mathrm{ng} / \mathrm{ml}(p<0.0001)$ as well as the resolution of myalgia in $92 \%$ of them [50]. A meta-analysis involving 2420 statin-treated patients from 7 studies provided evidence of an association between plasma vitamin $D$ levels and statin-associated myalgia [51]. In this meta-analysis significantly lower levels of vitamin D were found in patients with statin-associated myalgia in comparison to asymptomatic patients.

On the basis of these results, it has been hypothesized that it would be reasonable to screen patients with statin-induced myalgia and those who begin statin therapy for vitamin D insufficiency [49] and to begin vitamin $D$ supplementation in doses of 400-2000 IU in those with low vitamin D levels $(<32 \mathrm{ng} / \mathrm{ml})$ [52]. However, a large post hoc analysis of the Treating to New Targets trial [53], in which the incidence of myalgia was compared between patients with vitamin D deficiency $(<30 \mathrm{ng} / \mathrm{ml})$ and those with normal levels, failed to find any relationship ( $\mathrm{HR}=0.89,95 \% \mathrm{Cl}: 0.62-1.27 ; p=0.51)$.

It is a matter of debate whether vitamin D insufficiency potentiates statin-induced myalgia or statins contribute to vitamin D deficiency. The latter seems to be less likely due to the fact that atorvastatin therapy was shown to increase serum 25-OH D concentrations [54, 55]. Although the mechanism of this association is not known, it has been hypothesized that vitamin $D$ deficiency may reduce nuclear vitamin $D$ receptor linked gene transcription and concomitant synthesis of proteins required for the repair of the T-tubular system as well as for the prevention of subsarcolemmal rupture [31, 35, 52, 56]. Statins such as atorvastatin and simvastatin are metabolized by CYP3A4, having 25-hydroxylase activity [57]. It has been suggested that vitamin $D$ deficiency may be associated with 'preferential shunting' of CYP3A4 for vitamin D hydroxylation, which in consequence results in reduced CYP3A4 availability for statin metabolism and further in statin-induced toxicity [52]. However, further studies are necessary to reveal the exact mechanism of the relationship between vitamin D deficiency and statin-induced myalgia. A placebo-controlled, double-blind study is required to establish whether vitamin D supplementation lowers the risk for statin-associated myalgia and whether its normalization in vitamin $D$ deficient, statin-intolerant patients would facilitate re-introduction of statins without the subsequent risk of myositis-myalgia events.

\section{Coenzyme $\mathrm{Q}_{10}$}

Numerous studies have demonstrated that statins, including lovastatin [58, 59], simvastatin $[60,61]$ and pravastatin sodium $[59,60]$, decrease $\mathrm{CoQ}_{10}$ levels in blood. Atorvastatin treatment was shown to be associated with a rapid and substantial decrease of plasma $\mathrm{CoQ}_{10}$ concentrations after 14 days of therapy initiation, and this tendency was even more pronounced after 30 days of therapy [62]. Also, in a double-blind placebo-controlled study including patients treated with either $20 \mathrm{mg} /$ day of pravastatin or $20 \mathrm{mg} /$ day of simvastatin for one month, a significant reduction in $\mathrm{CoQ}_{10}$ concentration in blood was observed $(50 \%$ and $54 \%$, respectively) [60]. Gradual decrease of blood $\mathrm{CoQ}_{10}$ level associated with pravastatin sodium (20, 40 , and $80 \mathrm{mg} /$ day) and lovastatin (10, 20, and $40 \mathrm{mg} /$ day) reaching $80 \%$ of the baseline value in the case of pravastatin and $71 \%$ of the baseline value in the case of lovastatin was demonstrated in hypercholesterolemic patients [59]. Laaksonen et al. [61] also demonstrated decreased levels of muscle $\mathrm{CoQ}_{10}$ in healthy volunteers treated with $20 \mathrm{mg} /$ day of simvastatin for 4 weeks. In a place- 
bo-controlled trial $\mathrm{CoQ}_{10}$ supplementation had no effect on the prevalence and severity of myalgia, time to onset of pain, muscle strength, or aerobic performance, despite increased serum concentrations of $\mathrm{CoQ}_{10}$ in the treatment group [63]. Finally, a meta-analysis of 6 randomized clinical trials including 450 participants assessing the effect of statins on plasma $\operatorname{CoQ}_{10}$ concentrations demonstrated a significant reduction in plasma $\mathrm{CoQ}_{10}$ concentrations following statin treatment, which was independent of statin formulations, duration or dose [64].

The drop in serum $\mathrm{CoQ}_{10}$ levels during statin therapy may be due to its lipid solubility and transport in low-density lipoproteins [35, 65]. Moreover, insufficient dietary $\operatorname{CoQ}_{10}$ [66], inhibition of $\mathrm{CoQ}_{10}$ biosynthesis [67] and excessive consumption of $\mathrm{CoQ}_{10}$ [68] have been demonstrated to be responsible for the depletion of serum levels following statin therapy [64]. According to studies, statin-induced depletion of $\mathrm{CoQ}_{10}$ may be associated with defective activity of cell division and apoptosis [69], enhanced production of free radicals and subsequent damage of mitochondrial DNA [70], reduced mitochondrial oxidative phosphorylation capacity resulting in mitochondrial dysfunction [71], and the dysfunction or injury of skeletal muscle [72]. Bouitbir et al. [73] observed that in skeletal muscle of patients with statin-induced myopathy, statins induced high oxidative stress, responsible for transcriptional deactivation of mitochondrial biogenesis as well as mitochondrial dysfunctions. Also Sirvent et al. [74] revealed simvastatin-induced mitochondrial impairment resulting from inhibition of the respiratory chain complex I in human skeletal muscle. Coenzyme $\mathrm{Q}_{10}\left(\mathrm{CoQ}_{10}\right)$ seems to be the most feasible factor involved in statin myopathy $[8,73,75]$. $\operatorname{CoQ}_{10}$ (coenzyme $\mathrm{Q}_{10}$, ubiquinone) is a constituent of the mitochondrial electron transport system participating in oxidative phosphorylation. It is also the end product of the mevalonate pathway inhibited by statins [8]. Decreased $\mathrm{CoQ}_{10}$ levels could result in reduced rates of mitochondrial electron transport and ATP biosynthesis, resulting in myalgia/ myopathy. Statins probably decrease serum concentration of $\mathrm{CoQ}_{10}$ secondary to the reduction of low-density lipoprotein (LDL), which is the main carrier of this coenzyme in serum [76].

One theory concerning statin-associated myopathy suggests that the decrease in serum $\operatorname{CoQ}_{10}$ levels during statin therapy is associated mainly with the reduction in LDL particles, which transport $\mathrm{CoQ}_{10}$ particles $[35,77]$. Although muscle biopsies studies failed to identify reliable signs of reductions in muscle $\operatorname{CoQ}_{10}$ levels, in one study microscopic evidence of statin myopathy and reductions of $30 \%$ in intramuscular $\mathrm{CoQ}_{10}$ levels were observed in patients treated with simvastatin $80 \mathrm{mg}$ for 8 weeks [35]. The results of other studies assessing the effects of statins on $\mathrm{CoQ}_{10}$ levels in muscle suggest that the administration of statins does not reduce intramuscular $\mathrm{CoQ}_{10}$ concentrations, even in patients with symptomatic statin-induced myopathy [35].

Studies in which different transcriptional patterns between statin myopathic and statin-tolerant subjects after eccentric leg exercise were observed support the theory that statins can deleteriously affect mitochondrial function. Hubal et al. [78] demonstrated diminished skeletal muscle gene expression for oxidative phosphorylation-related and mitochondrial ribosomal protein genes in symptomatic subjects treated with a statin in comparison with asymptomatic subjects. Moreover, they revealed different mitochondrial gene expression before statin treatment (at baseline) between subjects who had tolerated and had not tolerated statin therapy in the past.

Studies analyzing the effects of $\mathrm{CoQ}_{10}$ supplementation on statin-induced myopathy gave conflicting results. A study in which patients treated with simvastatin were also administered $200 \mathrm{mg}$ / day $\mathrm{CoQ}_{10}$ or placebo for 12 weeks failed to confirm the effectiveness of coenzyme $Q$, since the frequency of myalgia was similar in both groups [79]. Also in the meta-analysis of Banach et al. [80] no significant effect of $\mathrm{CoQ}_{10}$ supplementation on statin-induced myopathy was seen. This finding was proved in the updated (2015) meta-analysis of randomized controlled trials, in which no significant benefits of $\mathrm{CoQ}_{10}$ supplementation in ameliorating statin-induced myopathy, even using higher $\mathrm{CoQ}_{10}$ doses, were observed [81]. However, in another study, a $40 \%$ decrease in pain severity and $38 \%$ in pain interference with daily activities was observed in patients supplemented with $100 \mathrm{mg} /$ day of $\mathrm{CoQ}_{10}$ [82]. Moreover, some studies demonstrated that $\mathrm{CoQ}_{10}$ supplementation diminished the symptoms of statin-induced myopathy, particularly when high doses (> $600 \mathrm{mg} / \mathrm{d}$ ) of $\mathrm{CoQ}_{10}$ were used $[35,80,83]$. Even if coenzyme $\mathrm{Q}_{10}$ administration does not relieve myalgia, it may be beneficial due to the fact that $\mathrm{CoQ}_{10}$ enhances adenosine- 5 -triphosphate production, exerts antioxidant activity, influences the expression of multiple genes, has membrane stabilizing properties, protects against LDL oxidation and inhibits proinflammatory cytokines and other factors [35, $80,84,85]$. Moreover, it has been suggested that the addition of $\mathrm{CoQ}_{10}$ to statin therapy may protect hepatocytes from statin-induced injuries and reduce their adverse effects $[45,80,86]$.

Mitochondrial dysfunction in patients with statin-induced myopathy and normal CK levels was also suggested by the picture of muscle biopsy 
including increased intramuscular lipid deposits, ragged red fibers and decreased cytochrome oxidase staining $[5,8,35]$. These abnormalities were reversed following the discontinuation of statin use $[5,8]$. Moreover, in skeletal muscle biopsies of patients treated with $80 \mathrm{mg} /$ day of simvastatin for 8 weeks, considerably diminished mitochondrial DNA levels were observed [87]. However, in biopsies of patients receiving $40 \mathrm{mg} /$ day of atorvastatin such alterations were not seen $[8,87]$. These findings suggest that statins may exert toxic effects on mitochondria, in this way being partially responsible for adverse effects on skeletal muscle [87]. Also, in a randomized controlled clinical trial [40] assessing muscle mitochondrial respiratory chain-enzyme complex activity, reduced total mitochondrial volume or fewer mitochondria per cell were observed in patients treated with simvastatin. Moreover, in patients on simvastatin, reduced mitochondrial mass was found. It has been suggested that high doses of simvastatin promote stress on skeletal myocytes, which in consequence results in the elimination of mitochondria and diminished levels of mtDNA and finally contributes to mitochondrial dysfunction [87].

In the study of Lamperti et al. [88], muscle structure was not disturbed in most patients with statin-related increases in CK levels. Studies assessing mitochondrial function on the basis of mitochondrial respiratory chain complex III activity or measurements of high-energy phosphate concentrations performed at rest in patients receiving statins revealed that mitochondrial function was not compromised $[8,88,89]$.

Several studies have also demonstrated that variations in the gene for coenzyme $Q$ synthase may also predispose to statin-induced myopathy. The presence of polymorphisms (rs4693075 and rs4693570) in the COQ2 gene was shown to increase the risk of myopathy in rosuvastatin- and atorvastatin-induced patients and the risk of myalgia in statin-treated individuals, respectively $[65,90]$. These single nucleotide polymorphisms (SNPs) are directly involved in CoQ deficiency, and this seems to be a mechanism for statin-induced myopathy $[35,91]$.

\section{SLCO1B1}

The Effectiveness of Additional Reductions in Cholesterol and Homocysteine (SEARCH) study demonstrated the relationship between the presence of a SNP located within the SLCO1B1 gene (chromosome 12) and simvastatin-related myopathy [16]. SLCO1B1 encodes organic anion-transporting polypeptide (OATP)1B1, which regulates the hepatic uptake of statins. SLCO1B1 has been implicated in statin-induced myopathy. Link et al. [16] observed a strong association between the occurrence of CK level $>3 \times$ ULN and $>5 \times$ baseline or muscle symptoms with CK $>10 \times$ ULN (myopathy) in patients treated with $80 \mathrm{mg} /$ day of simvastatin and presence of the non-coding SNP rs4363657. This SNP was suggested to be in nearly complete linkage disequilibrium with a non-synonymous SNP, 521T>C (rs4149056). The 521T/C transition (SLCO1B1*5) which encodes a valine to arginine amino acid substitution at residue 147 (V147L) was suggested to influence serum levels of statins and thus to increase the risk of myopathy. The frequency of statin-induced myopathy in individuals without the * 5 alleles was $19 \%, 27 \%$ in heterozygous individuals and $50 \%$ in ${ }^{*} 5 /{ }^{*} 5$ homozygotes [92]. Carr et al. [93] reported that the rs4149056 SNP in SLCO1B1 is related to statin-induced myopathy. In patients with the $C$ allele (CT/CC) the odds ratio (OR) for statin-induced myopathy (CK > 4x ULN) was 2.09 (1.27-3.45). The risk of severe myopathy (CK > 10× ULN/rhabdomyolysis) associated with the $\mathrm{C}$ allele is even greater, with an OR of 4.47 (1.84-10.84) [93]. In this study, 78\% of cases with myopathy were on simvastatin. When the analysis was restricted to those receiving simvastatin, a stronger relationship between SLCO1B1 521T/C and both myopathy $(O R=1.92(1.08-3.42)$ and severe myopathy $(\mathrm{OR}=4.99$ (1.72-14.50) was observed. Moreover, this association seems to be dose-related ( $\geq 40 \mathrm{mg} /$ day simvastatin), implying the importance of dose-genotype interaction. In the study of Carr et al. [93], no association between the studied SNP and atorvastatin-induced myopathy was observed. This result was confirmed by Brunham et al. [94]. Moreover, Niemi [95] demonstrated that in the case of simvastatin, the area under the curve (AUC) was 221\% higher in patients with the 521CC haplotype than in patients with the 521TT haplotype, while with atorvastatin treatment the mean increase in AUC was smaller (173\%). The relationship was insignificant with other statins. In the SEARCH study, the OR for the development of simvastatin-related myopathy was found to be 4.5 with the rs4363657 polymorphism (heterozygote) and 16.9 with 521CC (homozygote). Because this polymorphism is not associated with atorvastatin-induced musculoskeletal effects, it is hypothesized that the SLCO1B1 effects depend on the type of statin used [96]. Hydrophilic statins may be actively transported into hepatocytes expressing OATP, while lipophilic ones may be subjected to non-selective diffusion into extra-hepatic tissues, including skeletal muscle [97]. The PRIMO study revealed that the administration of pravastatin and fluvastatin, which are the most hydrophilic statins, is associated with the lowest 
risk of myalgia, whereas simvastatin (the most lipophilic statin) treatment is most likely to cause adverse effects on muscle. Another strongly lipophilic statin was withdrawn from the market due to high risk of rhabdomyolysis [8, 14].

\section{Cytochrome P enzyme (CYP)}

The cytochrome P enzyme (CYP) enzyme system is associated with phase 1 metabolism of various statins, and its isozymes - CYP3A4 and CYP2D6 - catalyze the majority of CYP-mediated drug metabolic reactions in humans [44, 98]. It has been demonstrated that polymorphisms within the CYP3A4/5, CYP2D6, and CYP2C9 genes can affect their rates of metabolism. Simvastatin is mainly metabolized by CYP2D6, while CYP3A4 and CYP3A5 metabolize atorvastatin. Mutations in CYP2D6 influence the rate of statin metabolism. On the basis of these genetic variations, patients can be divided into extensive metabolizers, poor metabolizers and ultra-rapid metabolizers [44, 99-101]. In theory, persons who are homozygous poor metabolizers have higher plasma levels of the statin and are at higher risk of its adverse effects [102]. In the study of Mulder et al. [102], in such persons the highest incidence of simvastatin intolerability (including myalgia and persistent elevation of creatine phosphokinase levels > 3 times the upper normal limit) was observed. Following atorvastatin therapy, homozygotes for the CYP3A $5^{\star} 3$ allele had higher serum CK levels compared to heterozygotes, which may indicate greater muscle damage among homozygotes [103]. It was also suggested that in homozygous patients with a frameshift mutation in CYP2C8 (475 delA) the risk of cerivastatin-induced rhabdomyolysis is increased due to the formation of mainly inactive protein unable to metabolize cerivastatin [104].

\section{ATP-binding cassette transporters subfamily $B$ (ABC transporters)}

The ATP-binding cassette subfamily B (ABCB) is responsible for liver drug efflux and mediates the final step in the hepatobiliary clearance of statins. The key function of proteins in this family is to limit the access of drugs to protected tissue compartments and to eliminate drugs and their metabolites via bile [105]. It has been suggested that SNPs within its genes, which are involved in both pharmacokinetic (ABCB1, ABCC2, ABCG2 and $A B C B 11$ ) and pharmacodynamic ( $A B C A 1$, $A B C G 5$ and $A B C G 8)$ properties of statins, may be associated with statin-induced intolerance [106, 107]. Fiegenbaum et al. [108] observed a greater reduction in total and LDL cholesterol after simvastatin treatment in carriers of the $1236 \mathrm{~T}$ variant (rs11285030) and $2677 \mathrm{G} / \mathrm{T}$ polymorphism (rs2032582) of the ABCB1 gene encoding the multidrug resistance protein MDR1. Another genotype-phenotype association study on a cohort of 116 hypercholesterolemic patients demonstrated that ABCB1 variants (1236T, 2677 non-G and $3435 T$ ) were less frequent in patients with adverse muscle effects [108, 109]. It has been found that the ABCB1 3435T allele is associated with impaired efflux and could enhance intestinal absorption of statins [110]. In carriers of the TTT haplotype, function of MDR1 was decreased, atorvastatin half-life was in these patients $24 \%$ longer than in subjects with the CGC haplotype $(p<0.05)$, and simvastatin acid and atorvastatin AUC was significantly larger [111]. Hoenig et al. [112] analyzed the influence of the C3435T polymorphism in ABCB1 and found that the $T$ allele was more frequent in patients with myalgia than those without it ( 0.80 vs. 0.62 ), while the $C$ allele was less frequent (0.20 vs. $0.38, p=0.043)$. Also, Ferrari et al. [113] reported that carriers of the ABCB1 C1236T SNP had an OR for statin-induced elevated serum CK levels of $4.67(p<0.05)$.

Another member of this family is breast cancer resistant protein (BCRP) (encoded by the ABCG2 gene), which modulates the absorption and elimination of statins, such as rosuvastatin and atorvastatin $[107,114,115]$. Alteration of the activity of other efflux proteins from the family of breast cancer resistance proteins (BCRP/ABCG2) further influences the pharmacokinetics of most statins, especially atorvastatin and rosuvastatin [116, 117]. The 421C/A SNP within the ABCG2 gene is associated with diminished BCRP transport activity and, in consequence, with reduced absorption of statins [118]. Other studies demonstrated a $144 \%$ increase in the AUC of rosuvastatin and $72 \%$ for atorvastatin in carriers of the ABCG2 421AA haplotype in comparison to the 421CC haplotype [116], and a $111 \%$ increase for simvastatin lactone due to reduced intestinal efflux of statins [117]. Polymorphisms within the genes of $A B C$ transporters G5 (ABCG5) and G8 (ABCG8), which are involved in intracellular cholesterol transport and mediate biliary excretion of cholesterol, may impair this pathway and alter cholesterol biosynthesis and response to statin treatment $[107,118$, 119]. Genotyping of the SLCO1B1, ABCB1 and ABCG2 genes may prove useful as a clinical approach improving statin safety.

\section{Carnitine palmitoyl-2 (CPT-2) deficiency}

The carnitine palmitoyltransferase (CPT) enzyme system, along with acyl-coenzyme A (CoA) synthetase and carnitine-acylcarnitine translocase (CACT), plays an essential role in the transfer of long chain fatty acids (LCFA) from the cytosol to the mitochondrial matrix $[120,121]$. CPT II de- 
ficiency, which is an autosomal recessive disorder, was first reported in 1973 by DiMauro et al. [122] in adults with exercise-induced rhabdomyolysis. In patients with CPT-2 deficiency, heavy exercise, cold exposure, infection, emotional stress or fasting is associated with the appearance of recurrent myalgia, rhabdomyolysis and myoglobinuria [121]. CPT-2 allows acyl Co-As into the mitochondrial matrix for $\beta$-oxidation of fatty acids, which are the major energy source for sustained skeletal muscle exercise [123]. Elevated excretion of acylcarnitine and an increased acylcarnitine/carnitine ratio in the plasma have been observed in CPT-2-deficient patients [124]. According to the study of Vladutiu et al. [125], the occurrence of this metabolic mutation occurs in $0.4 \%$ of the general population and in $3.8 \%$ of patients with statin myopathy. The frequency of heterozygotes for CPT-2 deficiency was 13-fold higher than that expected in the general population. Moreover, in their study, patient with homozygous CPT-2 deficiency developed progressive worsening of symptoms with cerivastatin therapy, and episodic rhabdomyolysis and myoglobinuria unrelated to exercise occurred even 5 years after discontinuing the statin. In heterozygotes, muscle-related symptoms resolved on discontinuation of the statin. All these observations indicate that statins can both provoke and worsen symptoms in individuals with CPT-2 deficiency [126].

\section{Farnesyl pyrophosphate and geranylgeranyl pyrophosphate}

Farnesyl pyrophosphate and geranylgeranyl pyrophosphate are intermediates in the HMG$\mathrm{COA}$ reductase pathway and end products of the mevalonate pathway. These two compounds normally activate regulatory guanosine 59-triphosphate-binding proteins, thus stimulating growth and maintenance of cells and hampering apoptosis [8]. The decrease in the level of farnesyl pyrophosphate and geranylgeranyl pyrophosphate has been suspected to be associated with adverse effects of statins due to the fact that statin-related morphologic changes and inhibition of protein synthesis in cultured rat skeletal muscle cells were reduced following the application of farnesol and geranylgeraniol. Moreover, specific inhibition of squalene epoxidase or squalene synthase (downstream compounds of cholesterol synthesis) resulted in the hampering of statin-induced apoptosis of human muscle cells $[8,127]$. It has been suggested that statin-induced myopathy is associated with the diminution of geranylgeranyl pyrophosphate metabolites but not with the inhibition of cholesterol synthesis [126].

According to Guijarro et al. [128], statin-induced myopathy may be associated with the stimulation of vascular smooth muscle cells apoptosis. Statins, which hamper the production of farnesyl pyrophosphate, inhibit the prenylation of GTP-binding proteins Ras, Rac and Rho, resulting in decreased levels of prenylated forms. Dysprenylation of these small GTPases leads to vacuolation of myofibers, degeneration and swelling of organelles and ultimately to apoptosis. Moreover, lamin dysprenylation raises cell susceptibility to mechanical stress $[129,130]$. This, in turn, leads to increased cytosolic calcium levels with subsequent activation of caspase-3 and caspase-9. These proteolytic enzymes play a key role in cell death. An in vivo study demonstrated the occurrence of statin-induced apoptosis of muscle cells which was inhibited by supplementation with the isoprenoids farnesyl pyrophosphate and geranylgeranyl pyrophosphate.

\section{Impaired calcium signaling}

Calcium $\left(\mathrm{Ca}^{2+}\right)$ release and uptake is critical for normal function of muscle cells. The results of some studies have suggested that statins may induce myopathy through the impairment of calcium signaling. Statins have been demonstrated to initiate the release of calcium from the sarcoplasmic reticulum through ryanodine receptors and to stimulate in human skeletal muscle long-lasting fura-2 calcium transients, which results in the activation of calpain and caspases 3 and 9 [127]. Moreover, the inhibition of calcium release via calcium chelation was shown to reverse this effect. Depolarization and calcium extrusion by the permeability transition pore and $\mathrm{Na}^{+} / \mathrm{Ca}^{2+}$ exchanger due to (a) impairment of mitochondrial respiration and (b) cytoplasmic and sarcoplasmic reticulum calcium overload leading to calcium waves seem to be the mechanisms responsible for the aforementioned effects [127]. The hypothesis of a role for calcium in statin-induced myopathy was supported by the results of muscle biopsies which demonstrated increased expression of ryanodine receptors 3 (RR3) and structural changes within the T-tubular system of the sarcoplasmic reticulum. However, it remains unknown whether increased RR3 expression is a risk factor of statin myopathy or a result of it $[131,132]$. Possible mechanisms of statin-induced side effects are presented in Figure 1.

In addition, the risk of statin-induced muscle-related symptoms may be associated with interactions of high doses of statins with concomitant drugs. According to studies, caution is advised during simultaneous use of high doses of statins and some other drugs. Table I (prepared on the basis of the review by Ahmad [133]) summarizes data on the safe use of statins and concomitant drugs. 
Molecular mechanisms of statin intolerance

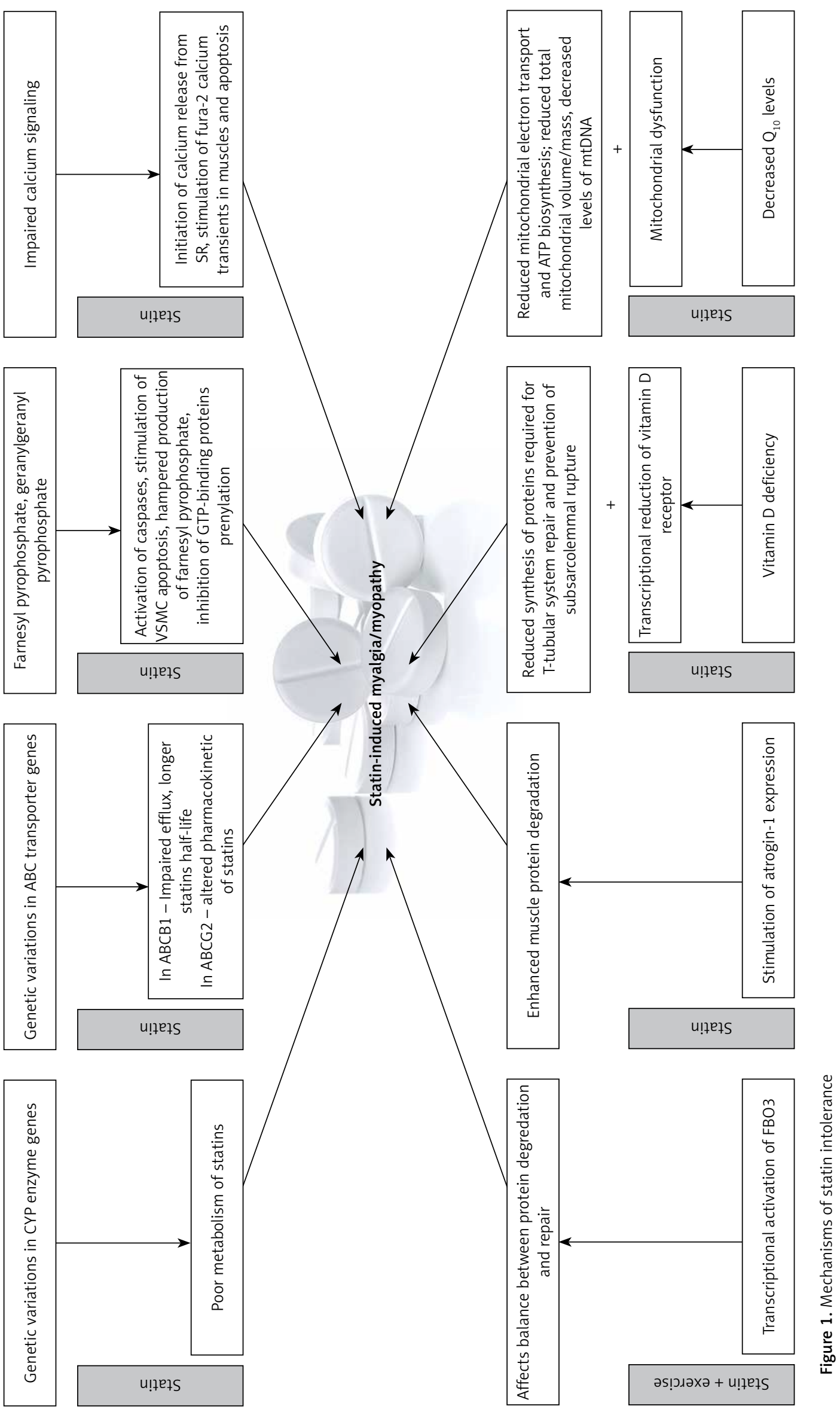


Table I. Recommendations for concomitant drug use (prepared on the basis of the review by Ahmad [133])

\begin{tabular}{|c|c|c|}
\hline Statin & Dose & Concomitant drug \\
\hline \multirow[t]{3}{*}{ Pravastatin } & $\leq 20 \mathrm{mg} /$ day & Cyclosporine \\
\hline & $\leq 40 \mathrm{mg} /$ day & Macrolide antibiotics \\
\hline & Any dose & $\begin{array}{l}\text { Antifungals, protease inhibitor, amiodarone, verapamil } \\
\text { and dilitiazem, amlodipine, ranolazine, dronedarone, } \\
\text { rifampin }\end{array}$ \\
\hline \multirow[t]{2}{*}{ Fluvastatin } & $\leq 20 \mathrm{mg} /$ day & Cyclosporine, antifungals \\
\hline & Any dose & $\begin{array}{c}\text { Macrolide antibiotics, protease inhibitor, amiodarone, } \\
\text { verapamil and dilitiazem, amlodipine, ranolazine, } \\
\text { dronedarone, rifampin }\end{array}$ \\
\hline \multirow[t]{2}{*}{ Rosuvastatin } & $\leq 5 \mathrm{mg} /$ day & Cyclosporine \\
\hline & Any dose & $\begin{array}{l}\text { Antifungals, macrolide antibiotics, protease inhibitor, } \\
\text { amiodarone, verapamil and dilitiazem, amlodipine, } \\
\text { ranolazine, dronedarone, rifampin }\end{array}$ \\
\hline \multirow[t]{2}{*}{ Atorvastatin } & $\leq 20 \mathrm{mg} /$ day & Antifungals, macrolide antibiotics, amlodipine \\
\hline & Any dose & $\begin{array}{c}\text { Amiodarone, verapamil and dilitiazem, ranolazine, } \\
\text { dronedarone, rifampin }\end{array}$ \\
\hline \multirow{3}{*}{$\begin{array}{l}\text { Simvastatin, amlodipine } \\
\text { (The dose of } 20 \text { mg of } \\
\text { simvastatin should not be } \\
\text { exceeded when used in } \\
\text { combination with amlodipine) }\end{array}$} & Any dose & Rifampin \\
\hline & $\leq 10 \mathrm{mg} / \mathrm{day}$ & Verapamil and dilitiazem, dronedarone \\
\hline & $\leq 20 \mathrm{mg} /$ day & Amiodarone, ranolazine \\
\hline \multirow[t]{2}{*}{ Lovastatin } & $\leq 40 \mathrm{mg} /$ day & Amiodarone \\
\hline & Any dose & Amlodipine, ranolazine, dronedarone, rifampin \\
\hline \multirow[t]{3}{*}{ Pitavastatin } & Any dose & $\begin{array}{l}\text { Antifungals, amiodarone, calcium antagonists, } \\
\text { dronedarone, protease inhibitors, ranolazine }\end{array}$ \\
\hline & $\leq 1 \mathrm{mg} /$ day & Macrolide antibiotics \\
\hline & $\leq 2 \mathrm{mg} / \mathrm{day}$ & Rifampin \\
\hline
\end{tabular}

\section{Conclusions}

As shown in this review, statin-induced muscle-related adverse events constitute a highly heterogeneous clinical disorder with numerous, complex etiologies. Muscle-related adverse events can be attributed to a variety of genetic backgrounds. Myopathy can be a manifestation of mitochondrial dysfunction, changes in enzyme and muscle protein metabolism, alterations in ion fluxes, autoimmune phenomena, and changes in cell membrane dynamics, among other things. Clearly, every patient who presents with statin-related side effects cannot undergo the type of exhaustive molecular characterization that would include all of these mechanisms. Frequently the only solution is to either discontinue the statin or attempt intermittent dosing strategies at a low dose. Among the most important potential causes of muscle-related adverse events with statin therapy are drug interactions [134]. Potentially treatable etiologies include thyroid and parathyroid dysfunction, electrolyte disturbances, and polymyalgia rheumatica. It would be beneficial to perform a prospective, randomized, placebo controlled trial of vitamin D supplementation in patients with statin-induced myalgia.

\section{Acknowledgments}

Three authors are (partially) supported by the Healthy Ageing Research Centre project (REGPOT2012-2013-1, 7FP).

\section{Conflict of interest}

The authors declare no conflict of interest.

\section{References}

1. Guyton JR, Bays HE, Grundy SM, TA Jacobson. An assessment by the Statin Intolerance Panel: 2014 update. J Clin Lipidol 2014; 8 (3 Suppl.): S72-81.

2. Banach M, Rizzo M, Toth PP, et al. Statin intolerance an attempt at a unified definition. Position paper from an International Lipid Expert Panel. Arch Med Sci 2015; 11: 1-23.

3. Banach M, Rizzo M, Toth PP, et al. Statin intolerance an attempt at a unified definition. Position paper from an International Lipid Expert Panel. Expert Opin Drug Saf 2015; 14: 935-55. 
4. Lanctôt KL, Naranjo CA. Comparison of the Bayesian approach and a simple algorithm for assessment of adverse drug events. Clin Pharmacol Ther 1995; 58: 692-8.

5. Phillips PS, Haas RH, Bannykh S, et al.; Scripps Mercy Clinical Research Center. Statin-associated myopathy with normal creatine kinase levels. Ann Intern Med 2002; 137: 581-5.

6. Thompson PD, Clarkson P, Karas RH. Statin-associated myopathy. JAMA 2003; 289: 1681-90.

7. Needham M, Fabian V, Knezevic W, Panegyres P, Zilko P, Mastaglia FL. Progressive myopathy with up-regulation of MHC-I associated with statin therapy. Neuromuscul Disord 2007; 17: 194-200.

8. Bitzur R, Cohen H, Kamari Y, Harats D. Intolerance to statins: mechanisms and management. Diabetes Care 2013; 36 Suppl. 2: S325-30.

9. Schalke BB, Schmidt B, Toyka K, Hartung HP. Pravastatin-associated inflammatory myopathy. N Engl J Med 1992; 327: 649-50.

10. Grable-Esposito P, Katzberg HD, Greenberg SA, Srinivasan J, Katz J, Amato AA. Immune-mediated necrotiz ing myopathy associated with statins. Muscle Nerve 2010; 41: 185-90.

11. Mammen AL, Chung T, Christopher-Stine L, et al. Autoantibodies against 3-hydroxy-3-methylglutaryl-coenzyme $A$ reductase in patients with statin-associated autoimmune myopathy. Arthritis Rheum 2011; 63: 713-21.

12. Banach M, Aronow WS, Serban C, et al. Lipids, blood pressure and kidney update 2014. Pharmacol Res 2015; 95-96: 111-25.

13. Bays H. Statin safety: an overview and assessment of the data 2005. Am J Cardiol 2006; 97: 6C-26C.

14. Bruckert E, Hayem G, Dejager S, Yau C, Bégaud B. Mild to moderate muscular symptoms with high-dosage statin therapy in hyperlipidemic patients the PRIMO study. Cardiovasc Drugs Ther 2005; 19: 403-14.

15. Law M, Rudnicka AR. Statin safety: a systematic review. Am J Cardiol 2006; 97: 52C-60C.

16. SEARCH Collaborative Group, Link E, Parish S, Armitage J, et al. SLCO1B1 variants and statin-induced myopathy - a genome wide study. N Engl J Med 2008; 359: 789-99.

17. Mancini GB, Baker S, Bergeron J, et al. Diagnosis, prevention, and management of statin adverse effects and intolerance: proceedings of a Canadian Working Group Consensus Conference. Can J Cardiol 2011; 27: 635-62.

18. Broncel M, Gorzelak-Pabiś P, Sahebkar A, et al. Lipid and Blood Pressure Meta-analysis Collaboration (LBPMC) Group. Sleep changes following statin therapy: a systematic review and meta-analysis of randomized placebo-controlled polysomnographic trials. Arch Med Sci 2015; 11: 915-26.

19. Guyton JR, Campbell KB, Lakey WC. Statin intolerance: more questions than answers. Expert Rev Clin Pharmacol 2014; 7: 1-3.

20. Aronow WS. Lipid-lowering therapy in older persons. Arch Med Sci 2015; 11: 43-56.

21. Banach M, Aronow WS, Serban MC, Rysz J, Voroneanu L, Covic A. Lipids, blood pressure and kidney update 2015. Lipids Health Dis 2015; 14: 167.

22. http://www.drugs.com/drug-interactions

23. Arca M, Pigna G. Treating statin-intolerant patients. Diabetes Metab Syndr Obes 2011; 4: 155-66.

24. Armitage J. The safety of statins in clinical practice. Lancet 2007; 370: 1781-90.
25. Cohen D, Anania F, Chalasani N. An assessment of statin safety by hepatologists. Am J Cardiol 2006; 97: 77C-81C.

26. Pasternak RC, Smith SC Jr, Bairey-Merz CN, Grundy SM, Cleeman JI, Lenfant C. ACC/AHA/NHLBI clinical advisory on the use and safety of statins. Circulation 2002; 106: 1024-8.

27. Davidson $M H$, Robinson JG. Safety of aggressive lipid management. J Am Coll Cardiol 2007; 49: 1753-62.

28. Kornbrust DJ, MacDonald JS, Peter CP, et al. Toxicity of the HMG coenzyme A reductase inhibitor, lovastatin, to rabbits. J Pharmacol Exp Ther 1989; 248: 498-505.

29. Dujovne CA. Side effects of statins: hepatitis versus "transaminitis"-myositis versus "CPKitis". Am J Cardiol 2002; 89: 1411-3.

30. Calderon RM, Cubeddu LX, Goldberg RB, Schiff ER. Statins in the treatment of dyslipidemia in the presence of elevated liver aminotransferase levels: a therapeutic dilemma. Mayo Clin Proc 2010; 85: 349-56.

31. Dale KM, White CM, Henyan NN, Kluger J, Coleman CI. Impact of statin dosing intensity on transaminase and creatine kinase. Am J Med 2007; 120: 706-12.

32. Molokhia M, McKeigue P, Curcin V, Majeed A. Statin induced myopathy and myalgia: time trend analysis and comparison of risk associated with statin class from 1991-2006. PLoS One 2008; 3: e2522.

33. Thompson PD, Clarkson PM, Rosenson RS; National Lipid Association Statin Safety Task Force Muscle Safety Expert Panel. An assessment of statin safety by muscle experts. Am J Cardiol 2006; 97: 69C-76C.

34 . Reiner Ž. Statins in the primary prevention of cardiovascular disease. Nat Rev Cardiol 2013; 10: 453-64.

35. Marcoff L, Thompson PD. The role of coenzyme Q10 in statin-associated myopathy: a systematic review. J Am Coll Cardiol 2007; 49: 2231-7.

36. Urso ML, Clarkson PM, Hittel D, Hoffman EP, Thompson PD. Changes in ubiquitin proteasome pathway gene expression in skeletal muscle with exercise and statins. Arterioscler Thromb Vasc Biol 2005; 25: 2560-6.

37. Thompson PD, Clarkson P, Karas RH. Statin-associated myopathy. JAMA 2003; 289: 1681-90.

38. Draeger A, Monastyrskaya K, Mohaupt M, et al. Statin therapy induces ultra-structural damage in skeletal muscle in patients without myalgia. J Pathol 2006; 210: 94-102.

39. Yokoyama M, Seo T, Park T, et al. Effects of lipoprotein lipase and statins on cholesterol uptake into heart and skeletal muscle. J Lipid Res 2007; 48: 646-55.

40. Paiva $H$, Thelen KM, Van Coster $R$, et al. High-dose statins and skeletal muscle metabolism in humans: a randomized, controlled trial. Clin Pharmacol Ther 2005; 78: 60-8.

41. Guis S, Figarella-Branger D, Mattei JP, et al. In vivo and in vitro characterization of skeletal muscle metabolism in patients with statin-induced adverse effects. Arthritis Rheum 2006; 55: 551-7.

42. Phillips PS, Haas RH. Statin myopathy as a metabolic muscle disease. Expert Rev Cardiovasc Ther 2008; 6: 971-8.

43. Mossman B, Behl C. Selenoprotein synthesis and side-effects of statins. Lancet 2004; 363: 892-4.

44. Ghatak A, Faheem O, Thompson PD. The genetics of statin-induced myopathy. Atherosclerosis 2010; 210: 337-43.

45. Jimenez-Santos MA, Juarez-Rojop IE. Tovilla-Zarate CA, et al. Coenzyme Q10 supplementation improves metabolic parameters, liver function and mitochondrial 
respiration in rats with high doses of atorvastatin and a cholesterol-rich diet. Lipids Health Dis 2014; 13: 22.

46. Hanai J, Cao P, Tanksale P, et al. The muscle-specific ubiquitin ligase atrogin-1/MAFbx mediates statin-induced muscle toxicity. J Clin Invest 2007; 117: 3940-51.

47. Siddals KW, Marshman E, Westwood M, Gibson JM. Abrogation of insulin-like growth factor-I (IGF-I) and insulin action by mevalonic acid depletion: synergy between protein prenylation and receptor glycosylation pathways. J Biol Chem 2004; 279: 38353-9.

48. Tintignac LA, Lagirand J, Batonnet S, Sirri V, Leibovitch MP, Leibovitch SA. Degradation of MyoD mediated by the SCF (MAFbx) ubiquitin ligase. J Biol Chem 2005; 280: 2847-56.

49. Lee P, Greenfield JR, Campbell LV. Vitamin D insufficiency: a novel mechanism of statin-induced myalgia? Clin Endocrinol (Oxf) 2009; 71: 154-5.

50. Ahmed W, Khan N, Glueck CJ, et al. Low serum $25(\mathrm{OH})$ vitamin $D$ levels $(<32 \mathrm{ng} / \mathrm{mL})$ are associated with reversible myositis-myalgia in statin-treated patients. Transl Res 2009; 153: 11-6.

51. Michalska-Kasiczak M, Sahebkar A, Mikhailidis DP, et al. Analysis of vitamin $D$ levels in patients with and without statin-associated myalgia. A systematic review and meta-analysis of 7 studies with 2420 patients. Int J Cardiol 2015; 178: 111-6.

52. Gupta A, Thompson PD. The relationship of vitamin D deficiency to statin myopathy. Atherosclerosis 2011 215: 23-9.

53. Bittner V, Wenger NK, Waters DD, et al. Vitamin D levels are not related to myalgias in statin-treated patients with stable coronary disease. J Am Coll Cardiol 2010; 55: A177.

54. Pérez-Castrillón JL, Vega G, Abad L, et al. Effects of Atorvastatin on vitamin $D$ levels in patients with acute ischemic heart disease. Am J Cardiol 2007; 99: 903-5.

55. Duell PB, Connor WE. Vitamin D deficiency is associated with myalgias in hyperlipidemic subjects taking statins. Circulation 2008; 118: S470 (Abstract 3701).

56. Schubert L, Deluca HF. Hypophosphatemia is responsible for skeletal muscle weakness of vitamin D deficiency. Arch Biochem Biophys 2010; 500: 157-61.

57. Gupta RP, He YA, Patrick KS, et al. CYP3A4 is a vitamin D-24- and 25-hydroxylase: analysis of structure function by site-directed mutagenesis. J Clin Endocrinol Metabol 2005; 90: 1210-9.

58. Folkers K, Langsjoen P, Willis R, et al. Lovastatin decreases coenzyme Q levels in humans. Proc Natl Acad Sci U S A 1990; 87: 8931-4.

59. Mortensen SA, Leth A, Agner E, Rohde M. Dose-related decrease of serum coenzyme Q10 during treatment with $\mathrm{HMG}-\mathrm{CoA}$ reductase inhibitors. Mol Aspects Med 1997; 18 (Suppl): S137-44.

60. Ghirlanda G, Oradei A, Manto A, et al. Evidence of plasma CoQ10-lowering effect by HMG-CoA reductase inhibitors: a double-blind, placebo-controlled study. J Clin Pharmacol 1993; 33: 226-9.

61. Laaksonen R, Jokelainen K, Sahi T, et al. Decreases in serum ubiquinone concentrations do not result in reduced levels in muscle tissue during short-term simvastatin treatment in humans. Clin Pharmacol Ther 1995; 57: 62-6.

62. Rundek T, Naini A, Sacco R, Coates K, DiMauro S. Atorvastatin decreases the coenzyme Q10 level in the blood of patients at risk for cardiovascular disease and stroke. Arch Neurol 2004; 61: 889-92.

63. Taylor BA, Lorson L, White CM, Thompson PD. A randomized trial of coenzyme Q10 in patients with con- firmed statin myopathy. Atherosclerosis 2015; 238: $329 e 335$.

64. Banach M, Serban C, Ursoniu S, et al. Statin therapy and plasma coenzyme Q10 concentrations. A systematic review and meta-analysis of placebo-controlled trials. Pharmacol Res 2015; 99: 329-36.

65. Ruaño G1, Windemuth $A, W u A H$, et al. Mechanisms of statin-induced myalgia assessed by physiogenomic associations. Atherosclerosis 2011; 218: 451-6.

66. Desbats MA, Lunardi G, Doimo M, Trevisson E, Salviati L. Genetic bases and clinical manifestations of coenzyme Q10 (CoQ 10) deficiency. J Inherit Metab Dis 2015; 38: 145-56.

67. Littarru GP, Langsjoen P. Coenzyme Q10 and statins: biochemical and clinical implications, Mitochondrion 2007; 7: S168-74.

68. Yubero D, Montero R, Artuch R, Land JM, Heales SJ, Hargreaves IP. Biochemical diagnosis of coenzyme q10 deficiency. Mol Syndromol 2014; 5: 147-55.

69. Hodges S, Hertz N, Lockwood K, Lister R. CoQ10: could it have a role in cancer management? BioFactors 1999; 9: 365-70.

70. Deichmann R, Lavie C, Andrews S. Coenzyme Q10 and statin-induced mitochondrial dysfunction. Ochsner J 2010; 10: 16-21.

71. Larsen S, Stride N, Hey-Mogensen M, et al. Simvastatin effects on skeletal muscle: relation to decreased mitochondrial function and glucose intolerance. J Am Coll Cardiol 2013; 61: 44-53.

72. Fukami M, Maeda N, Fukushige J, et al. Effects of HMGCoA reductase inhibitors on skeletal muscles of rabbits. Res Exp Med 1993; 193: 263-73.

73. Bouitbir J, Charles AL, Echaniz-Laguna A, et al. Opposite effects of statins on mitochondria of cardiac and skeletal muscles: a 'mitohormesis' mechanism involving reactive oxygen species and PGC-1. Eur Heart J 2012; 33: 1397-407.

74. Sirvent P, Bordenave S, Vermaelen M, et al. Simvastatin induces impairment in skeletal muscle while heart is protected. Biochem Biophys Res Commun 2005; 338: 1426-34.

75. Banach M, Mikhailidis DP; Lipid and Blood Pressure Meta-analysis Collaboration (LBPMC) Group. In reply - Coenzyme Q10 and statin-induced myopathy. Mayo Clin Proc 2015; 90: 420-1.

76. Schaars CF, Stalenhoef AF. Effects of ubiquinone (coenzyme Q10) on myopathy in statin users. Curr Opin Lipidol 2008; 19: 553-7.

77. Parker BA, Thompson PD. Effect of statins on skeletal muscle: exercise, myopathy, and muscle outcomes. Exerc Sport Sci Rev 2012; 40: 188-94.

78. Hubal MJ, Reich KA, De Biase A, et al. Transcriptional deficits in oxidative phosphorylation with statin myopathy. Muscle Nerve 2011; 44: 393-401.

79. Young JM, Florkowski CM, Molyneux SL, et al. Effect of coenzyme Q (10) supplementation on simvastatininduced myalgia. Am J Cardiol 2007; 100: 1400-3.

80. Banach M, Serban C, Sahebkar A, et al. Effects of coenzyme Q10 on statin-induced myopathy: a meta-analysis of randomized controlled trials. Mayo Clin Proc 2015; 90: 24-34.

81. Banach M, Serban C, Amirhossein S, et al. Futility of supplementation with coenzyme Q10 for statin-induced myopathy: an updated (2015) meta-analysis of randomized controlled trials. Eur Heart J 2015; 36 (suppl. 1): 1047 
82. Caso G, Kelly P, McNurlan MA, Lawson WE. Effect of coenzyme q10 on myopathic symptoms in patients treated with statins. Am J Cardiol 2007; 99: 1409-12.

83. Thibault A, Samid D, Tompkins AC, et al. Phase I study of lovastatin, an inhibitor of the mevalonate pathway, in patients with cancer. Clin Cancer Res 1996; 2: 483-91.

84. Schmelzer C, Lindner I, Vock C, Fujii K, Doring F. Functional connections and pathways of coenzyme Q10 inducible genes: an in-silico study. IUBMB Life 2007; 59: 628-33.

85. Groneberg DA, Kindermann B, Althammer $M$, et al. Coenzyme Q10 affects expression of genes involved in cell signalling, metabolism and transport in human CaCo-2 cells. Int J Biochem Cell Biol 2005; 37: 1208-18.

86. Eghbal MA, Abdoli N, Azarmi Y. Efficiency of hepatocyte pretreatment with coenzyme Q10 against statin toxicity. Arh Hig Rada Toksikol 2014; 65: 101-8.

87. Schick BA, Laaksonen R, Frohlich J), et al. Decreased skeletal muscle mitochondrial DNA in patients treated with high-dose simvastatin. Clin Pharmacol Ther 2007; 81: 650-3.

88. Lamperti C, Naini AB, Lucchini V, et al. Muscle coenzyme Q10 level in statin-related myopathy. Arch Neurol 2005; 62: 1709-12.

89. Laaksonen R, Jokelainen K, Laakso J, et al. The effect of simvastatin treatment on natural antioxidants in low-density lipoproteins and high-energy phosphates and ubiquinone in skeletal muscle. Am J Cardiol 1996; 77: 851-4.

90. Puccetti L, Ciani F, Auteri A. Genetic involvement in statins induced myopathy. Preliminary data from an observational case-control study. Atherosclerosis 2010; 211: 28-9.

91. Golomb BA, Evans MA. Statin adverse effects: a re view of the literature and evidence for a mitochondrial mechanism. Am J Cardiovasc Drugs 2008; 8: 373-418.

92. Voora D, Shah SH, Spasojevic I, et al. The SLCO1B1*5 genetic variant is associated with statin-induced side effects. J Am Coll Cardiol 2009; 54: 1609-16.

93. Carr DF, O’Meara H, Jorgensen AL, et al. SLCO1B1 genetic variant associated with statin-induced myopathy: a proof-of-concept study using the clinical practice research data link. Clin Pharmacol Ther 2013; 94: 695-701.

94. Brunham LR, Lansberg PJ, Zhang L, et al. Differential effect of the rs4149056 variant in SLCO1B1 on myopathy associated with simvastatin and atorvastatin. Pharmacogenomics J 2012; 12: 233-7.

95. Niemi M. Transporter pharmacogenetics and statin toxicity. Clin Pharmacol Ther 2010; 87: 130-3.

96. Santos PC, Gagliardi AC, Miname MH, et al. SLCO1B1 haplotypes are not associated with atorvastatin-in duced myalgia in Brazilian patients with familial hypercholesterolemia. Eur J Clin Pharmacol 2012; 68: 273-9.

97. Neuvonen PJ, Niemi M, Backman JT. Drug interactions with lipid-lowering drugs: mechanisms and clinical relevance. Clin Pharmacol Ther 2006; 80: 565-81.

98. Butler MA, Lang NP, Young JF, et al. Determination of CYP1A2 and NAT2 phenotypes in human populations by analysis of caffeine urinary metabolites. Pharmacogenetics 1992; 2: 116-27.

99. Marez D, Legrand M, Sabbagh N, et al. Polymorphism of the cytochrome P450 CYP2D6 gene in a European population: characterization of 48 mutations and 53 alleles, their frequencies and evolution. Pharmacogenetics 1997; 7: 193-202.
100. Johansson I, Lundqvist E, Bertilsson L, et al. Inherited amplification of an active gene in the cytochrome P450 CYP2D locus as a cause of ultrarapid metabolism of debrisoquine. Proc Natl Acad Sci USA 1993; 90: 11825-9.

101. Dahl ML, Johansson I, Bertilsson L, Ingelman-Sundberg $M$, Sjoqvist F. Ultrarapid hydroxylation of debrisoquine in a Swedish population. Analysis of the molecular genetic basis. J Pharmacol Exp Ther 1995; 274: 516-20.

102. Mulder AB, van Lijf HJ, Bon MA, et al. Association of polymorphism in the cytochrome CYP2D6 and the efficacy and tolerability of simvastatin. Clin Pharmacol Ther 2001; 70: 546-51.

103. Wilke RA, Moore JH, Burmester JK. Relative impact of CYP3A genotype and concomitant medication on the severity of atorvastatin-induced muscle damage. Pharmacogenet Genom 2005; 15: 415-21.

104. Ishikawa C, Ozaki H, Nakajima T, et al. A frameshift variant of CYP2C8 was identified in a patient who suffered from rhabdomyolysis after administration of cerivastatin. J Hum Genet 2004; 49: 582-5.

105. Reiner Z. Resistance and intolerance to statins. Nutr Metab Cardiovasc Dis 2014; 24: 1057-66.

106. Sirtori CR, Mombelli G, Triolo M, Laaksonen R. Clinical response to statins: mechanism(s) of variable activity and adverse effects. Ann Med 2012; 44: 419-32.

107. Duman I. Role of pharmacogenetics on response to statins: a genotype-based approach to statin therapy outcome. J Cardiol Therapy 2014; 1: 111-20.

108. Fiegenbaum M, da Silveira FR, Van der Sand CR, Van der Sand LC, Ferreira ME, Pires RC. The role of common variants of $A B C B 1, C Y P 3 A 4$, and CYP3A5 genes in lipid-lowering efficacy and safety of simvastatin treatment. Clin Pharmacol Ther 2005; 78: 551-8.

109. Feng QP, Wilke RA, Baye TM. Individualized risk for statin-induced myopathy. Current knowledge, emerging challenges, and potential solutions. Pharmacogenomics 2012; 13: 579-94.

110. Peters BJ, Klungel $\mathrm{OH}$, de Boer A, Maitland-van der Zee $\mathrm{AH}$. Genetic determinants of response to statins. Expert Rev Cardiovasc Ther 2009; 7: 977-83.

111. Keskitalo JE, Kurkinen KJ, Neuvoneni PJ, Niemi M. $A B C B 1$ haplotypes differentially affect the pharmacokinetics of the acid and lactone forms of simvastatin and atorvastatin. Clin Pharmacol Ther 2008; 84: 457-61.

112. Hoenig MR, Walker PJ, Gurnsey C, Beadle K, Johnson L. The C3435T polymorphism in ABCB1 influences atorvastatin efficacy and muscle symptoms in a high-risk vascular cohort. J Clin Lipidol 2011; 5: 91-6.

113. Ferrari M, Guasti L, Maresca A, et al. Association between statin-induced creatine kinase elevation and genetic polymorphisms in SLCO1B1, ABCB1 and ABCG2. Eur J Clin Pharmacol 2014; 70: 539-47.

114. Robey RW, To KK, Polgar O, et al. ABCG2: a perspective. Adv Drug Deliv Rev 2009; 61: 3-13.

115. Giorgi MA, Caroli C, Arazi HC, Di Girolamo G. Pharmacogenomics and adverse drug reactions: the case of statins. Expert Opin Pharmacother 2011; 12: 1499-509.

116. Keskitalo JE, Zolk O, Fromm MF, Kurkinen KJ, Neuvonen PJ, Niemi M. ABCG2 polymorphism markedly affects the pharmacokinetics of atorvastatin and rosuvastatin. Clin Pharmacol Ther 2009; 86: 197-203.

117. Keskitalo JE, Pasanen MK, Neuvonen PJ, Niemi M. Different effects of the ABCG2 c.421C >A SNP on the pharmacokinetics of fluvastatin, pravastatin and simvastatin. Pharmacogenomics 2009; 10: 1617-24. 
118. Rodrigues AC. Efflux and uptake transporters as determinants of statin response. Expert Opin Drug Metab Toxicol 2010; 6: 621-32.

119. Kajinami K, Brousseau ME, Ordovas JM, Schaefer EJ. Interactions between common genetic polymorphisms in ABCG5/G8 and CYP7A1 on LDL cholesterol-lowering response to atorvastatin. Atherosclerosis 2004; 175: 287-93.

120. Bieber LL. Carnitine. Ann Rev Biochem 1988; 57: 261-83.

121. Sigauke E, Rakheja D, Kitson K, Bennett MJ. Carnitine palmitoyltransferase II deficiency: a clinical, biochemical, and molecular review. Lab Invest 2003; 83: 1543-54.

122. DiMauro S, DiMauro PMM. Muscle carnitine palmitoyltransferase deficiency and myoglobinuria. Science 1973; 182: 929-31.

123. Harrison's principles of internal medicine. McGraw-Hill, New York 2009.

124. Bonnefont JP, Djouadi F, Prip-Buus C, et al. Carnitine palmitoyltransferases 1 and 2: biochemical, molecular and medical aspects. Mol Aspects Med 2004; 25: 495-520.

125. Vladutiu GD, Simmons Z, Isackson PJ, et al. Genetic risk factors associated with lipid-lowering drug-induced myopathies. Muscle Nerve 2006; 34: 153-62.

126. Flint OP, Masters BA, Gregg RE, Durham SK. Inhibition of cholesterol synthesis by squalene synthase inhibitors does not induce myotoxicity in vitro. Toxicol Appl Pharmacol 1997; 145: 91-8.

127. Sirvent P, Mercier J, Lacampagne A. New insights into mechanisms of statin-associated myotoxicity. Curr Opin Pharmacol 2008; 8: 333-8.

128. Guijarro C, Blanco-Colio LM, Ortego M, et al. 3-Hydroxy-3-methylglutaryl coenzyme A reductase and isoprenylation inhibitors induce apoptosis of vascular smooth muscle cells in culture. Circ Res 1998; 83: 490-500.

129. Vaklavas C, Chatzizisis YS, Ziakas A, et al. Molecular basis of statin-associated myopathy. Atherosclerosis 2009; 202: 18-28.

130. Harper CR, Jacobson TA. The broad spectrum of statin myopathy: from myalgia to rhabdomyolysis. Curr Opin Lipidol 2007; 18: 401-8.

131. Mohaupt MG, Karas RH, Babiychuk EB, et al. Association between statin-associated myopathy and skeletal muscle damage. CMAJ 2009; 181: E11-8.

132. Abd TT, Jacobson TA. Statin-induced myopathy: a review and update. Expert Opin Drug Saf 2011; 10: 373-87.

133. Ahmad Z. Statin intolerance. Am J Cardiol 2014; 113: 1765-71.

134. Mampuya WM, Frid D, Rocco M, et al. Treatment strategies in patients with statin intolerance: the Cleveland Clinic experience. Am Heart J 2013; 166: 597-603. 